\title{
ZONA ORGANIZADORA DE NUCLÉOLO E SUA RELAÇÃO COM A EPIDEMIOLOGIA DE CADELAS COM NEOPLASIA MAMÁRIA
}

\author{
(Nucleolar organization zone and its relationship with the epidemiology breast cancer in \\ bitches)
}

Louanne Affonso Montserrat, Diego Cassiano-Camuzi, Jankerle Neves Boeloni, Louisiane de Carvalho Nunes, Leonardo Oliveira Trivilin ${ }^{1}$

\author{
${ }^{1}$ Correspondência: louaffonso@hotmail.com
}

\begin{abstract}
RESUMO: As neoplasias mamárias são comumente encontradas em cadelas, sendo observada, geralmente, em animais senis, não castrados e sem predisposição racial. A técnica de AgNOR pode auxiliar no diagnóstico e prognóstico de tumores mamários. Objetivou-se relacionar o número de AgNOR aos dados epidemiológicos como idade de acometimento, raça do animal, status reprodutivo, status vivo ou morto, diagnóstico, recidiva e metástase. Fez-se um levantamento de dados de 49 cadelas com neoplasia mamária durante o período de 2011 a 2015 da rotina do HOVET-UFES e a técnica de AgNOR foi empregada em lâminas de tumores mamários de cadelas. Observou-se média de idade de 10 anos e contagem média de AgNOR correspondente a 4,42. As cadelas SRD foram as mais frequentes com média de AgNOR 4,22. O status vivo correspondeu a $63,64 \%$ das cadelas e a média de AgNOR foi 4,23, e as mortas, 36,36\% com média de AgNOR de 4,75. Encontrou-se 90,90\% de cadelas castradas, com média de AgNOR de 4,31 e não castradas 9,09\%, com média de AgNOR 5,48. Neoplasias malignas foram observadas em 40 (81,63\%) cadelas cuja média de AgNOR foi 4,46, e as benignas em nove $(18,37 \%)$ com média de AgNOR de 4,24. Das cadelas, $42(85,71 \%)$ não apresentaram recidiva tumoral, cuja média de AgNOR foi de 4,39, e em duas que apresentaram metástase tumoral, média de AgNOR de 5,04. Concluiu-se que o AgNOR varia em relação a idade e raça, e que também não houve dependência do status de sobrevida em relação ao número de $\mathrm{AgNOR}$, bem como em relação ao status reprodutivo. Ainda, que o número de AgNOR variou conforme o diagnóstico e foi maior em tumores malignos e quando houve metástase.
\end{abstract}

Palavras-chave: AgNOR; cães; dados epidemiológicos; tumor de mama

ABSTRACT: Breast cancer is commonly found in dogs, generally observed in senile and intact animals without breed predisposition. The AgNOR technique can aid in the diagnosis and prognosis of mammary tumors. Our objective was to correlate the number of AgNOR with epidemiological data such as age of onset, breed, reproductive status, live or death status, diagnosis, recurrence and metastasis. The clinical information was collected from HOVET-UFES routine of 49 bitches with mammary neoplasia from 2011 to 2015 and AgNOR technique was applied in bitches breast tumors slides. There was an average age of 10 years and average score of AgNOR corresponding to 4.42. Bitches of no specific breed most frequent, averaging AgNOR 4.22. The living status, corresponded to $63.64 \%$ of dogs and AgNOR average was 4.23; the dead 36.36\% with a mean AgNOR 4.75. Spayed bitches were $90.90 \%$, with AgNOR average of 4.31 and intact were $9.09 \%$, with AgNOR average 5.48. Malignancies were observed in 40 (81.63\%) bitches whose average AgNOR was 4.46 , and benign in nine (18.37\%) with a mean AgNOR of 4.24 .42 bitches $(85.71 \%)$ showed no tumor recurrence and average AgNOR was 4.39; two showed tumor metastasis with mean AgNOR of 5.04. It concluded that AgNOR varies in age and race. There was no dependence on survival status in relation to the number of AgNOR, as well as in relation to reproductive status. The number of AgNOR varied according to the diagnosis and was greater in malignancies tumors and when metastasis was present.

Key Words: AgNOR; breast cancer; dog; epidemiological data 


\section{INTRODUÇÃO}

Neoplasias

mamárias

são

comuns em cães, sendo encontradas em maior frequência nas cadelas (Oliveira Filho et al., 2010), cujo perfil é representado por animais idosos, não castrados e com várias nodulações (Varallo et al., 2012). Podem ocorrer em fêmeas de diferentes raças, porém, a maior incidência ocorre naquelas de pequeno porte (Sleeckx et al., 2011). De acordo com De Nardi et al. (2008) as principais raças acometidas por neoplasias mamárias são Poodles, Pastores Alemães, Cocker Spaniels, e também os animais sem raça definida.

Sorenmo et al., (2011) consideram a idade com um dos principais fatores para o surgimento de neoplasia em tecido mamário de cadelas. Porém, acrescenta-se a esses fatores de risco, a raça, dieta, obesidade, hormônios, cicloxigenase-2 e aspectos moleculares envolvidos na carcinogênese (Betz et al., 2012).

A raça, idade e status reprodutivo, bem como o histórico de terapia com progestágeno, ciclos estrais, pseudogestações, lactações e infecções do tecido mamário, partos, além de exame físico, citopatológico e histopatológico, compreendem o diagnóstico de neoplasia mamária em cadelas (Sorenmo, 2003; Cassali et al., 2011).

Ao longo dos últimos anos, o "método de AgNOR" foi aplicado em patologia tumoral, tanto para diagnóstico como para fins de prognóstico. As regiões organizadoras de nucléolo (AgNORs) são definidas como componentes nucleolares que contém um conjunto de proteínas argirofílicas, que são seletivamente coradas pelo método de prata (Trerè, 2000). Em estudo realizado por Bostock et al. (1992), avaliando prognóstico de tumor mamário, foi identificado uma relação do aumento da contagem de AgNOR com o aumento do grau de malignidade destes tumores.

Considerando que os dados relativos ao estudo molecular do tumor mamário canino estão longe de serem completos, pois o conhecimento de vias moleculares possui um potencial significativo para complementar e aperfeiçoar a abordagem diagnóstica e terapêutica em casos de tumor mamário canino (Klopfleisch et al., 2011). Ainda, que a zona organizadora de nucléolo abrange o estudo molecular e oferece uma boa avaliação da proliferação celular, ser de fácil manipulação e baixo custo (Morita et al., 1991; LÖHR et al.,1997). Questionamos se a contagem das AgNORs estaria relacionada a fatores epidemiológicos que afetam o prognóstico das cadelas com neoplasia mamária.

Desta forma, objetivou-se com este estudo avaliar a contagem de AgNORs e sua relação com dados epidemiológicos como, idade de acometimento, raça do animal, status reprodutivo, status vivo ou morto, diagnóstico, recidiva e metástase referentes às cadelas com neoplasia mamária.

\section{MATERIAL E MÉTODOS}

Foram utilizados 49 casos de neoplasia mamária em cadelas atendidas na rotina do Hospital Veterinário do Centro de Ciências Agrárias da Universidade Federal do Espírito Santo entre os anos de 2011 a 2015. Os casos em questão foram submetidos à mastectomia radical e os tumores encaminhados para exame histopatológico no Laboratório de Patologia Animal do Hospital Veterinário.

Após o levantamento dos casos do livro de registro do Hospital Veterinário, foram selecionadas as lâminas e os blocos de parafina correspondentes a cada caso, de onde foram obtidos novos cortes histológicos 
com $3 \mu \mathrm{m}$ de espessura para a aplicação da técnica histoquímica de AgNOR. Além disso, foram coletados dados epidemiológicos como idade, raça, status vivo ou morto, status reprodutivo, diagnóstico, recidiva tumoral e metástase da ficha de cada animal registrado no Hospital Veterinário e também por consulta aos proprietários.

A técnica de AgNOR correspondeu a metodologia modificada de Ploton et al. (1986). Em seguida os cortes foram lavados em água deionizada, desidratados em concentrações crescentes de álcool e montadas em resina sintética.

Para definir a frequência de AgNOR, foram contados os pontos marrom-acastanhados no interior dos núcleos em 100 células epiteliais tumorais, distribuídas em cinco campos aleatórios, em objetiva de 100x com o auxílio do óleo de imersão. A frequência AgNOR/núcleo de cada tumor mamário avaliado foi obtida dividindo- se número total de AgNOR por 100 (Kravis et al.,1996).

A partir dos dados da contagem obteve-se a média de AgNOR que foram submetidos à análise estatística descritiva. A mediana dos valores de AgNORs foi obtida para avaliar a dependência do status de vida ao número de AgNORs por núcleo celular pelo Teste Qui-quadrado, e a dependência do número de AgNORs aos status reprodutivo foi avaliada pelo Teste Exato de Fisher. Um teste de correlação de Pearson foi conduzido para avaliar o número de AgNORs com a idade.

\section{RESULTADOS}

A idade média das cadelas deste estudo foi de 10,1 anos e o número médio de AgNORs encontrado foi de $4,42 \pm 1,27$. A idade mais frequente observada nesse estudo foi de 12 anos com um número médio de 4,28 $\pm 1,41$ AgNORs. Das 49 cadelas estudadas, constatou-se a ocorrência de apenas uma cadela na idade de cinco anos e uma na idade de 15 anos que apresentaram, respectivamente, 2,4 e 7,23 AgNORs por núcleo celular. Além disso, o teste de correlação apontou uma interação fraca positiva $(r=0,23$, $\mathrm{P}<0,05)$ entre a progressão da idade e 0 aumento do número médio de AgNORs.

Em relação à raça a Tabela 1 mostra o número e o percentual de animais pertencentes a cada raça deste estudo, bem como a média de AgNOR encontrada em cada uma delas.

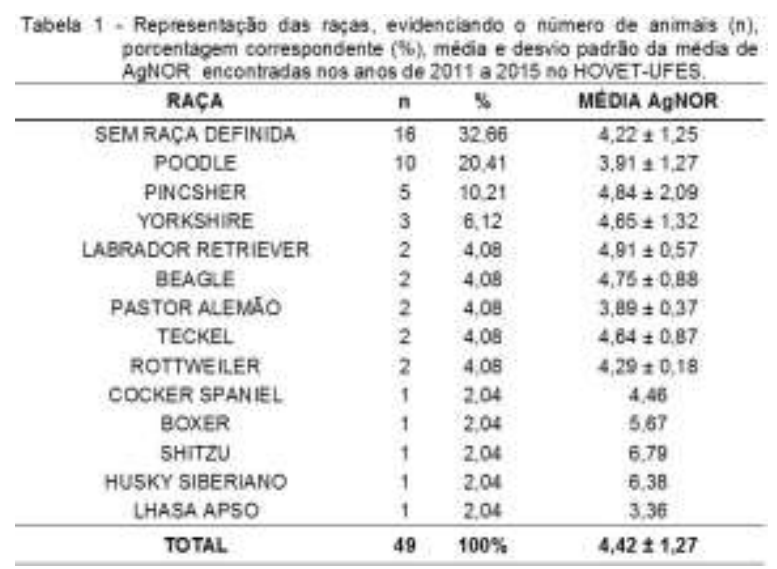

O estudo das AgNORs e sua dependência com status de vida das cadelas deste estudo foi conduzida em $44 \quad(89,8 \%)$ das 49 cadelas selecionadas. As outras cinco $(10,2 \%)$ cadelas não foram avaliadas por não ser possível obter informações junto aos proprietários, se estavam vivas ou mortas. A Tabela 2 mostra 0 agrupamento por ano de cirurgia, número de animais, porcentagem, o status de vida e o número médio de AgNORs quanto ao status de vida. 


\begin{tabular}{|c|c|c|c|c|c|c|}
\hline \multirow{2}{*}{$\begin{array}{l}\text { ANO DA } \\
\text { CIRURGIA }\end{array}$} & \multirow{2}{*}{ N } & \multirow{2}{*}{$*$} & \multicolumn{2}{|c|}{ STATUS DE VIDA } & \multicolumn{2}{|c|}{ MEDUA AGNOR } \\
\hline & & & vivo & MORTO & VIVAS & $\begin{array}{c}\text { MORTA } \\
\mathrm{s}\end{array}$ \\
\hline 2011 & 9 & 20,45 & $4(44,4 \%)$ & $5(55.5 \%)$ & $3,48=0,73$ & $\begin{array}{c}4,70 \pm \\
0.99\end{array}$ \\
\hline 2012 & 8 & 18,98 & $4(505)$ & $4(5000)$ & $3,94 \pm 0,93$ & $\begin{array}{c}5,12 t \\
1,09\end{array}$ \\
\hline 2013 & 15 & 34,09 & $10\{00,7 \%\}$ & $5(33,3 \%)$ & $4,49=1,23$ & $\begin{array}{l}5,35 \pm \\
1,72\end{array}$ \\
\hline 2014 & 10 & 22,73 & $8(80 \%)$ & $2(20 \%)$ & $4,07 \pm 0,90$ & $\begin{array}{c}2,62 \pm \\
0,59\end{array}$ \\
\hline 2015 & 2 & 4,54 & $2(100 \%)$ & - & $5,66+269$ & - \\
\hline TOTAL & 44 & $99,99 \%$ & $28(53,64 \% 6)$ & $16(36,36 \%)$ & $4,23 \pm 1,19$ & $\begin{array}{c}4,75 \pm \\
1,44\end{array}$ \\
\hline
\end{tabular}

No ano de 2011 o tempo de sobrevida foi de 26,4 meses, ao passo que no ano de 2012 foi de 18 meses. Em 2013 o tempo de sobrevida foi de 10,8 meses e em 2014 foi de quatro meses. A partir da Tabela 2 foi possível verificar uma tendência à redução do percentual de mortes das cadelas, mesmo que, os casos mais antigos, apresentem uma maior propensão a morrerem mais rápidos por serem mais idosos. Essas mortes ocorreram, segundo informações dos proprietários, por causas diferentes à neoplasia mamária, com exceção de duas cadelas que apresentaram metástase.

Um teste de dependência entre o número médio de AgNORs e o status de vida foi conduzido, a partir do agrupamento, entre os casos avaliados, em $\geq 4,17$, e $<4,17$, que correspondeu à mediana dos valores de AgNORs. Conforme demonstrado na Tabela 3, não houve diferença significativa, portanto, o status de vida do animal não foi dependente do número médio de AgNORs encontrado.

Tabela 3 - Relaçăo da dependència do status de sobrevida e contagem média de AgNOR em cadelas com tumor de mama entre os anos de 2011 a 2015 nO HOVET-UFES.

\begin{tabular}{cccc}
\hline STATUS DE & \multicolumn{3}{c}{ Valor de P } \\
SOBREVIDA & $=4,17$ & $<4,17$ & \\
\hline VIVO & 13 & 15 & 0 \\
MORTO & 11 & 5 & \\
\hline
\end{tabular}

Quanto ao status reprodutivo, obteve-se informação da ficha clínica e junto aos proprietários de 44 (89,79\%) cadelas, enquanto que em cinco
$(10,21 \%)$ não foi possível avaliá-lo. Das cadelas cujo status reprodutivo era conhecido, $40(90,90 \%)$ delas não eram castradas no momento da cirurgia e neste grupo foi possível observar um número médio de AgNORs de 4,31 \pm 1,25 , ao passo que as cadelas castradas antes da mastectomia eram de apenas quatro $(9,09 \%)$ animais, cuja média de AgNORs foi $5,48 \pm 1,49$. Além disso, não houve dependência do número médio de AgNORs com o status reprodutivo castrada ou não castrada $(P<0,05)$.

Dos 49 casos selecionados, 40 $(81,63 \%)$ tiveram diagnóstico de tumores malignos e o número médio de AgNORs foi $4,46 \pm 1,32$; e nove $(18,37 \%)$ neoplasias benignas, com número médio de AgNORs de 4,24 \pm 1,07. A Tabela 4 mostra o diagnóstico em cadelas com tumor de mama, o número de animais (n), a porcentagem e o número médio de AgNORs encontrados.

\begin{tabular}{|c|c|c|c|}
\hline DIAGNOSTICO & n & $\%$ & MEDIA AgNOR \\
\hline ADENOMA & 2 & 4,08 & $4,30 \pm 0,19$ \\
\hline ADENOMA COMPLEXO & 1 & 2,04 & 3,08 \\
\hline ADENOMA SECRET ORIO & 1 & 2,04 & 3,60 \\
\hline ADENOMA TUBULAR & 3 & 6,12 & $4.60 \pm 1,69$ \\
\hline TUMOR MISTO BENIGNO & 2 & 4.08 & $4.56 \pm 1,06$ \\
\hline ADENOCARCINOMA & 2 & 4.08 & $3.06 \pm 0.56$ \\
\hline CARCINOMA ADENOESCAMOSO & 1 & 2,04 & 6,38 \\
\hline CARCINOMA COMPLEXO & 15 & 30.62 & $4.52=1.19$ \\
\hline CARCINOMA EM TUMOR MISTO & 4 & 8,16 & $4.51 \pm 1.50$ \\
\hline CARCINOMA SIMPLES & 5 & 10.21 & $5,01=1,94$ \\
\hline CARCINOMA SOLIDO & 3 & 6,12 & $3,77 \pm 1,29$ \\
\hline CARCINOMA TUBULAR & 9 & 18,37 & $4,06 \pm 0,67$ \\
\hline OSTEOSSARCOMA & 1 & 2,04 & 7,23 \\
\hline TOTAL & 49 & $100 \%$ & $4,42 \pm 1,27$ \\
\hline
\end{tabular}

Das cadelas estudadas, 42 $(85,71 \%)$ não apresentaram recidiva tumoral, cuja média de AgNORs foi de $4,39 \pm 1,31$. Além disso, duas cadelas $(4,08 \%)$ foram diagnosticadas com metástase tumoral, tendo o número médio de AgNORs de 5,04 \pm 0,89. Cinco animais $(10,20 \%)$ não foram possíveis conhecer se tiveram recidivas ou metástase. 


\section{DISCUSSÃO}

Segundo Queiroga e Lopes (2002), a maioria das cadelas afetadas com neoplasia mamária tem idade entre os 8 e 10 anos de idade, podendo ocorrer tumores malignos em animais com menos de cinco anos. Neste estudo as cadelas apresentaram uma média de idade de 10,1 anos corroborando Toríbio et al. (2012) e Morris e Dobson (2007) que encontraram, média de idade de 10,17 e 10 anos, respectivamente. Desta forma, observase que ocorrência de neoplasias mamárias em cadelas abrange uma variação elevada em relação à idade, sendo encontradas em maior frequência em cadelas idosas.

Os dados referentes à contagem média de AgNOR do presente estudo assemelham-se aos dados encontrados por Sarli et al. (2002) onde o número de AgNORs foi de 4,32, independente do diagnóstico, em cadelas com tumor mamário que apresentaram idade média de 10 anos. Porém, diferem dos achados de Vaz-Curado et al. (2008) que avaliaram AgNOR em tumor mamário em cadelas encontrando uma média de idade de 9,13 anos, e número médio de AgNORs de 1,79, também independente do diagnóstico. Assim, observa-se uma variação no número de AgNORs encontrados em idades semelhantes, mesmo não havendo correlação entre a idade e número de AgNOR, que podem ser dependentes de outros fatores.

Um estudo realizado por Toríbio et al. (2012) com 336 cadelas constatou uma maior incidência de neoplasia mamária nas raças Poodle, Pastor Alemão, e cães SRD, respectivamente. Queiroga e Lopes (2002) afirmaram que não existe uma predisposição racial. Porém, neste estudo os cães Sem Raça Definida foram os mais encontrados, seguido de Poodle, Pinsher e outras raças. Essa diferença de raça, provavelmente, está relacionada ao número de animais distribuídos nas diferentes localidades que se baseiam os estudos de neoplasias mamárias em cadelas. No entanto, os resultados deste estudo permeiam os relatos de Sleeckx et al. (2011) que afirmaram serem as raças de pequeno porte as mais incidentes para neoplasia mamária.

No que diz respeito ao número de AgNOR por raça, não foram encontrados dados na literatura consultada que servissem de base para a discussão dos achados deste estudo. No entanto, permeando achados de AgNOR em tumores diferentes daqueles encontrados na glândula mamária de cadelas, observa-se a variação da contagem de AgNORs entre as raças, como é o caso do estudo realizado por Bostock et al. (1989) relacionando raças de cães com a média de AgNOR em caso de mastocitoma canino. Os autores constataram que em tumores bem diferenciados, para a raça Boxer o número de AgNOR foi de 1,5, Labrador/Retriever foi de 1,4, outras raças 1,3 e SRD 1,7.

A variação na contagem de AgNORs em neoplasias mamárias deste estudo, em relação à raça, mostra que outros fatores interferem no seu aparecimento, uma vez que as raças cuja frequência foi maior, diferiram em termos de número de AgNORs daquelas menos frequentes. Isso pode ser explicado pelo fato do AgNOR estar relacionado ao nível de transcrição celular, ciclo celular, ploidia e proliferação (Underwood e Giri, 1988; Trerè et al. 1989; Scheer e Benavente,1990), e portanto mais inerente à célula em questão do que à origem racial do animal.

Em relação à sobrevida de cadelas com neoplasia mamária, Daleck et al. (1998) relataram que de 23 animais submetidos ao estudo, 18 apresentaram taxa de sobrevida superior a 360 dias sem recidivas ou 
metástase. Segundo Misdorp (2002), animais com tumores mamários sobrevivem por até um ou dois anos após mastectomia. Os dados obtidos neste estudo se assemelham aos apresentados anteriormente $\mathrm{e}$, ainda assim, observa-se uma redução do número de mortes ao longo dos anos, provavelmente, devido a melhores condições de vida nas quais as cadelas estão inseridas nos tempos atuais, como também, mais opções de tratamento e detecção precoce da neoplasia mamária.

Pich et al. (2000) em revisão bibliográfica mostraram uma correlação significativa entre a alta quantidade de AgNOR e baixa sobrevida em humanos com diferentes tipos tumorais. Bostock et al. (1992) estudando 39 cadelas com carcinoma mamário, observou que os animais nos quais apresentavam a média de AgNORs abaixo de 8, tinham um prognóstico favorável e maior sobrevida, diferente de animais com contagem acima de 8 , que morreram antes de completar um ano de cirurgia. Contudo, observa-se que a alta quantidade de AgNOR encontrada, indicando uma alta atividade celular em tumores, apresentou associação com um prognóstico desfavorável e uma sobrevida menor em animais como também em humanos.

Um estudo realizado por Sacks et al. (1992) não encontraram relação entre a contagem de AgNOR e intervalo livre da doença ou sobrevida de tumores de mama em humanos. No caso das cadelas, o presente estudo também revelou não haver relação significativa entre o número médio de AgNORs e a sobrevida das mesmas. Ainda assim, é necessário adquirir dados de sobrevida de animais com câncer de mama, uma vez que é importante apresentar uma expectativa de vida para os proprietários dos mesmos.

Ainda no que tange a sobrevivência de cadelas com neoplasia mamária, Sarli et al. (2002), em estudo feito com tumores mamários em 60 cadelas, revelaram que 14 delas morreram, sendo que seis foram submetidas à eutanásia e oito morreram espontaneamente devido a disseminação do tumor. No presente estudo, os animais cujo status de vida foi morto, morreram de causas não relacionadas à doença específica, e somente duas devido à metástase distante. Ainda assim, quando cadelas apresentam tumores mais invasivos têm uma maior incidência de disseminação do tumor e desenvolvimento de metástase.

O status reprodutivo é um fator de risco para o desenvolvimento de neoplasia mamária em cadelas, e um estudo realizado por Ramos (2011) mostrou que $96 \%$ das cadelas acometidas com tumor de mama não eram castradas. Outros estudos também apontam o status não castrada ao risco de desenvolvimento de tumor mamário, como os descritos por Oliveira et al. (2003), Toríbio et al. (2012) e Salas et al. (2015).

Houve uma diferença entre a contagem de AgNORs nas cadelas castradas e não castradas, sendo que nessas últimas o número foi menor do que o observado para as primeiras. No entanto, o percentual de cadelas não castradas superou, e muito, a de castradas, o que poderia influenciar na contagem de AgNORs, uma vez que os dados dos mesmos foram descritos em média. No entanto, novos estudos se fazem necessários com um maior número de cadelas castradas para se relacionar com a contagem de AgNOR.

De acordo com Queiroga e Lopes (2002), aproximadamente $50 \%$ dos tumores de mama em cadelas são malignos. No entanto, os dados encontrados neste estudo diferem dos apresentados e se assemelham aos descritos por Toríbio et al. (2012) e Guim (2011) que encontraram tumores 
malignos em maior proporção em relação aos benignos.

Estudos realizados em diferentes tipos tumorais demostraram que as células malignas apresentam, frequentemente, uma maior quantidade de AgNOR do que células não malignas (Trerè, 2000). Jelesijević et al. (2003) e Bundgaard-Andersen et al. (2008) analisando tumores mamários em cães também encontraram número maior de AgNOR em relação a malignidade dos tumores. Os dados do presente estudo se assemelham aos dados apresentados, e provavelmente, o número de AgNORs encontrado em tumores benignos foi menor devido ao fato de terem sido encontrados em menor frequência em relação aos tumores malignos das cadelas estudadas. No entanto, nenhuma dependência foi observada entre 0 número de AgNORs e o tipo tumoral maligno ou benigno.

Os dados publicados por VazCurado et al. (2008) analisando contagem média de AgNORs em tumores benignos e malignos, mostraram 2,14 $\pm 0,54$ AgNORs em carcinoma tubular e 1,95 $\pm 0,30$ em carcinomas sólidos, concluindo não haver diferença significativa entre os tumores malignos. Porém, os achados do presente estudo mostraram-se diferentes e com número de AgNORs superior aos relatados anteriormente.

Carvalho (2012) analisando 168 cadelas com tumores mamários encontrou casos de recidiva em $36 \%$ delas, e a metástase foi observada em $12,3 \%$ das cadelas estudadas. Neste estudo, não se observou recidivas nas cadelas e apenas duas apresentaram metástase, cujo número de AgNOR observado foi maior que média encontrada.

A recidiva pode indicar uma falha técnica em relação ao planejamento terapêutico ou um tipo tumoral mais agressivo (Amar et al., 2005). No presente estudo não foram encontradas cadelas com recidivas, provavelmente, devido ao padrão terapêutico empregado no HOVET-UFES, onde se realiza mastectomia radical da cadeia mamária acometida, sendo a técnica indicada por Morris e Dobson (2007) onde analisaram que mastectomia radical é mais rápida e fácil de ser realizada.

Em tecidos neoplásicos a expressão de AgNOR foi encontrada para ser estritamente relacionado a duplicação celular (Trerè, 2000). Quando um tumor é infiltrativo ou invasivo a tendência a ocorrer metástase é maior (Morris e Dobson, 2007). Um estudo feito por Bhatt et al. (2013) com lesões mamárias em humanos constatou uma diferença de tumores malignos com metástase, tendo uma contagem alta de AgNORs, significativo de malignidade. No presente estudo os dados das cadelas com metástase apresentaram número de AgNOR acima da média encontrada. Assim, esse aumento de AgNOR, provavelmente, está associado ao aumento da proliferação celular de um tumor maligno que poderá culminar em metástase. No entanto, são necessários maiores estudos com um número maior de animais com metástase correlacionando-os ao AgNOR.

Observamos que o AgNOR relaciona-se de forma variável com os dados epidemiológicos de cadelas com câncer de mama, e que pesquisas agregando ferramentas biomoleculares, - AgNOR e dados epidemiológicos melhorariam de forma considerável as questões diagnósticas, terapêuticas e prognósticas de cadelas acometidas por neoplasias mamárias.

\section{CONCLUSÃO}

Concluiu-se que o AgNOR varia em relação a idade e raça, e que também não houve dependência do status de sobrevida em relação ao número de AgNOR, bem como em 
relação ao status reprodutivo. Ainda, que o número de AgNOR variou conforme o diagnóstico e foi maior em tumores malignos e quando houve metástase.

\section{REFERÊNCIAS}

AMAR, A.; CURIONI, O. A; FRANZI, S. A.; RAPOPORT, A. Recidivas locais após tratamento cirúrgico do carcinoma epidermóide de cabeça e pescoço em estágio avançado. Revista Colégio Brasileiro de Cirurgiões, v.32, n.2, p.60-63, 2005.

BETZ, D.; SCHOENROCK, D.; MISCHKEL, R.; BAUMGÄRTNER, W.; NOLTE, I. Postoperative treatment outcome in canine mammary tumors Multivariate analysis of the prognostic value of pre- and postoperatively available information. Tierärztliche Praxis Kleintiere, v.40, p. 235-242, 2012.

BHATT, J.; SHRIMALI, G.; PATEL, T. et al. Silver stained nucleolar organizer region count (AgNOR count) - Very useful tool in breast lesions. National Journal of Medical Research, v.3, n.3, p. 280-282, 2013.

BOSTOCK, D. E.; MORIARTY, J.; CROCKER, J. Correlation between histologic diagnosis mean nucleolar organizer region count and prognosis in canine mammary tumors. Veterinary Pathology, v.29, p.381-385, 1992.

BOSTOCK, D. E.; CROCKER, J.; HARRIS, K. et al. Nucleolar organizer regions as indicators of post-surgical prognosis in canine spontaneous mast cell tumors. British Journal of Cancer, v.59, p.915-918, 1989.

BUNDGAARD-ANDERSEN, K.; FLAGSTAD, A.; JENSEN, A. L. et al. Correlation between the Histopathological Diagnosis by AgNOR Count and AgNOR Area in Canine Mammary Tumours. Journal of Veterinary Internal Medicine, v.22, p.1174-1180, 2008.

CARVALHO, C. F. C. F. de. Neoplasias mamárias dos carnívoros domésticos - Estudo retrospectivo. 2012. Vila Real, 61 f. Dissertação (Mestrado em Ciências Veterinárias), Universidade de Trás-os-Montes e Alto Douro.

CASSALI, G. D.; LAVALLE, G. E.; DE NARDI, A. B. et al. Consensus for the Diagnosis, Prognosis and Treatment of Canine Mammary Tumors. Brazilian Journal of Veterinary Pathology, v.4, n.2, p.153-180, 2011.

DALECK, C. R.; FRANCESCHINI, P. H.; ALESSI, A. C. et al. Aspectos clínicos e cirúrgicos do tumor mamário canino. Ciencia Rural, v.28, n.1, p.95-100, 1998.

DE NARDI, A. B.; RODASKI, S.; ROCHA, N. S. et al. Neoplasias Mamárias. In: DALECK, R. D., NARDI, A. B. de., RODASKI, S. Oncologia em cães e gatos. São Paulo: Roca, 2008, Cap.25, p.371-383.

GUIM, T. N. Determinação de fatores prognósticos para tumores mamários caninos. 2011. Pelotas, 99 f. Tese (Doutorado em Patologia Animal) - Programa de Pós Graduação em Veterinária, Universidade Federal de Pelotas.

JELESIJEVIĆ, T.; JOVANOVIĆ, M.; KNEZEVIĆ, M. et al. Quantitative and qualitative analysis of AgNOR in benign and malignant canine mammary gland tumours. Acta Veterinaria Belgrade, v.53, n.5-6, p.353-360, 2003.

KLOPFLEISCH, R.; EULER, H. von; SARLI, G. et al. Molecular carcinogenesis of canine mammary tumors: news from an old disease. Veterinary Pathology, v.48, p.98-116, 2011.

KRAVIS, L. D.; VAIL. D. M.; KISSEBERTH, W. C. et al. Frequency of argyrophilic nucleolar organizer regions in fine-needle aspirates and biopsy specimens from mast cell tumors in dogs. Journal of the American Veterinary Medical Association. v.209, p.1418-1420, 1996.

LÖHR, C. V.; TEIFKE, J. P.; FAILING, K. et al. Characterization of the proliferation state in canine mammary tumors by the standardized AgNOR method with postfixation and immunohistologic of Ki-67 and PCNA. Veterinary Pathology, v.34; p.212-221, 1997.

MISDORP, W. Tumors of the Mammary Gland. In: MEUTEN, D. J. Tumors in Domestic Animals. 4. ed. Estados Unidos: John Wiley \& Sons, 2002, Cap. 12, p.575-606.

MORRIS, J.; DOBSON, J. Oncologia em Pequenos Animais. São Paulo: Roca Ltda, 2007, Cap.12, p.185-192.

MORITA, M.; KUWANO, H.; MATSUDA, H. et al. Prognostic significance of argyrophilic nucleolar organizer regions in esophageal carcinoma. Cancer Research, v.51, n.19, p.5339-5341, 1991.

OLIVEIRA, L. O. de; OLIVEIRA, R. T. de; LORETTI, A. P.; RODRIGUES, R.; DRIEMEIER, D. Aspectos epidemiológicos da neoplasia mamária canina. Acta Scientiae Veterinariae, v.31, p.105-110, 2003.

OLIVEIRA FILHO, J. C. KOMMERS, G. D. MASUDA, E. K. et al. Estudo retrospectivo de 1647 tumores mamários em cães. Pesquisa 
Veterinária Brasileira, v.30, n.2, p.177-185, 2010.

$\mathrm{PICH}, \quad$ A.; CHIUSA, L.; MARGARIA, E. Prognostic relevance of AgNORs in tumor pathology. Micron, v.31, p.133-141, 2000.

PLOTON, D.; MENAGER, M.; JEANNESSON, $P$. et al. Improvement in the staining and in the visualisation of the argyrophilic proteins of the nucleolar organizer region at the optical level. Histochemical Journal, v.18, p.5-14, 1986.

QUEIROGA, F.; LOPES, C. Tumores mamários caninos - novas perspectivas. In: CONGRESSO DE CIÊNCIAS VETERINÁRIAS= PROCEEDINGS OF THE VETERINARY SCIENCES CONGRESS, 2002, Anais...Oeiras, Portugual [S.I.]: SPVC, 2002. p. 183-190.

RAMOS, C. S. Associação entre os fatores epidemiológicos e neoplasias mamárias em cadelas. 2011. Jaboticabal, 48 f. Dissertação (Mestrado em Cirurgia Veterinária) - Faculdade de Ciências Agrárias e Veterinárias, Universidade Estadual Paulista.

SACKS, N. P.; ROBERTSON, J. F.; ELLIS, I. O. et al. Silver-stained nucleolar organizer region counts are of no prognostic value in primary breast cancer. European Journal of Surgical Oncology, v.18, p.98-102, 1992.

SALAS, Y.; MÁRQUEZ, A.; DIAZ, D. et al. Epidemiological study of mammary tumors in female dogs diagnosed during the period 20022012: A growing animal health problem. Research Article, v.10, n.5, p.1-15, 2015.

SARLI, G.; PREZIOSI R.; BENAZZI, C.; CASTELLANI, G. et al. Prognostic value of histologic stage and proliferative activity in canine malignant mammary tumors. Journal of Veterinary Diagnostic Investigation, v.14, p.2534, 2002.

SCHEER, U.; BENAVENTE, R. Functional and dynamic aspects of the mammalian nucleolus. Bioessays, v.12, n.1, p.14-21, 1990.

SLEECKX, N.; DE ROOSTER, H.; VELDHUIS KROEZE, E. J. B. et al. Canine mammary tumours, an overview. Reproduction in Domestic Animals, v.46, p.1112-1131, 2011.

SORENMO, K. Canine mammary gland tumors. Veterinary Clinics: Small Animal Practice, v.33, p.573-596, 2003.

SORENMO, K. U.; RASOTTO, R.; ZAPPULLI, V. et al. Development, anatomy, histology, lymphatic drainage, clinical features, and cell differentiation markers of canine mammary gland neoplasms. Veterinay Pathology, v.48, n.1, p.8597, 2011.
TORÍBIO, J. M. de M. L.; LIMA, A. E.; MARTINS FILHO, E. F. et al. Caracterização clínica, classificação histopatológica e geoprocessamento das neoplasias mamárias em cadelas de Salvador, Bahia. Revista Ceres, v.59, n.4, p.427-433, 2012.

TRERÉ, D. AgNOR staining and quantification. Elsevier, v.31, p.127-131, 2000.

TRERÈ, D.; PESSION, A.; DERENZINI, M. The silver-stained proteins of interphasic nucleolar organizer regions as a parameter of cell duplication rate. Experimental Cell Research, v,189, p.131-137, 1989.

UNDERWOOD, J. C. E.; GIRI, D. D. Nucleolar organizer regions as diagnostic discriminants for malignancy. The Journal of Pathology, v.155, p. 95-96, 1988

VARALLO, G. R.; RAPOSO, T. M. M.; TERRA, E. M. et al. Neoplasias mamárias: idade ao diagnóstico, raça e cadeia mamária mais acometida em cadelas atendidas no HV da UNESP, Jaboticabal, SP. In: Oncovet, 7, 2012, João Pessoa. Anais...do VII Oncovet, 2012. p. 20-22.

VAZ-CURADO, A. P.; GUERRA, J. L.; DIAS, R. A. Estudo quantitativo e morfológico das regiões organizadoras de nucléolo coradas pela prata (AgNORs) em neoplasias benignas e malignas da glândula mamária da espécie canina. Brazilian Journal of Veterinary Research and Animal Science, v.45, n.3, p.206-210, 2008. 